\title{
Structure Study of Solution Formed Poly(3-hexylthiophene) Nanofibers
}

John D. Roehling, Adam J. Moulé, and Ilke Arslan

Department of Chemical Engineering and Materials Science, University of California, Davis, One Shields Ave., Davis, CA 95616

Carbon-free energy sources are quickly becoming a necessity for the modern world economy; energy usage is expected to double from $15 \mathrm{TW}$ to $30 \mathrm{TW}$ by 2050 . Solar energy offers the only renewable solution that can realistically meet the growing demand for energy without resorting to burning fossil fuels. Organic photovoltaics (OPVs) lead the way with the lowest cost per watt potential, capable of competing with fossil fuels economically, but the efficiency has to be increased to warrant their widespread usage. It has been shown that the efficiency can be increased by using nano-scale phase separated mixtures of donor and acceptor materials [poly(3-hexylthiophene) (P3HT) and $\mathrm{C}_{61}$-butyric acid methyl ester (PCBM) respectively], known as a bulk-heterojunction (BHJ) device [1]. These small donor/acceptor domains allow for efficient charge separation, a major limiting factor in the efficiency of OPV devices, but little is known about these domains' fine structure. A fundamental study is difficult to perform on BHJs directly due to low resolution, but devices with similar efficiencies can also be fabricated from pre-made solution processed P3HT nanofibers [2]. Electron tomography studies of the BHJ have shown the P3HT forms fibular networks with domain sizes on the order of the solution formed nanofibers $(\sim 15-25 \mathrm{~nm})$ [3], suggesting that the P3HT domains in BHJs may be very similar to the solution formed nanofibers. Therefore, studying the P3HT nanofibers should provide similar information about the structure of P3HT domains in a BHJ. Through the use of the transmission electron microscope (TEM) and electron diffraction, the structure of solution processed nanofibers has been studied in detail, and for the first time high-resolution (HRTEM) images of single P3HT nanofibers have been obtained. From these studies it has been shown that the polymer chains do not always stack as previously demonstrated, perpendicular to nanofiber length [4], but can stack parallel to the nanofiber length also. Until now, solution formed nanofibers have been assumed to always have perpendicular chain stacking $[2,5]$. The parallel stacked nanofibers shown here are very crystalline; the $(020)$ spacing between chains ( $\pi$ stacking direction) is reduced from $0.38 \mathrm{~nm}$ to $0.35 \mathrm{~nm}$ in these parallel stacked fibers, indicating stronger bonding in this direction. Figure 1 shows two HRTEM images of parallel stacked P3HT nanofibers. Figure 2 shows selected area diffraction (SAD) patterns and images of parallel stacked nanofibers, confirming the (020) spacing and the stacking direction of the polymer chains seen in the high-resolution images. Differences in crystallinity are seen in the perpendicularly stacked fibers between this study and the literature, and this discrepancy is found to be due to a difference in solvents used to form the nanofibers. A study of different solvents used to form the nanofibers and a comparison of their structures will be discussed.

References

[1]A.J. Moulé, K. Meerholz, Adv. Mater., 20, (2008), 240

[2] S. Berson, R. De Bettignies, S. Bailly, and S. Guillerez, Adv. Funct. Mater. 17, (2007), 1377

[3] S. van Bavel, E. Sourty, G. de With, K. Frolic, J. Loos, Macromolecules, 42, (2009), 7396

[4] K. J. Ihn, J. Moulton, P. Smith, J. of Polymer Sci. Part B-Polymer Phys., 31, (1993), 735 
[5] S. Samitsu, T. Shimomura, S. Heike, T. Hashizume, and K. Ito, Macromolecules, 41, (2008), 8000

[6] This work is supported by the UC Fee Money Grant. The aid of Dr. Nigel Browning is gratefully acknowledged.

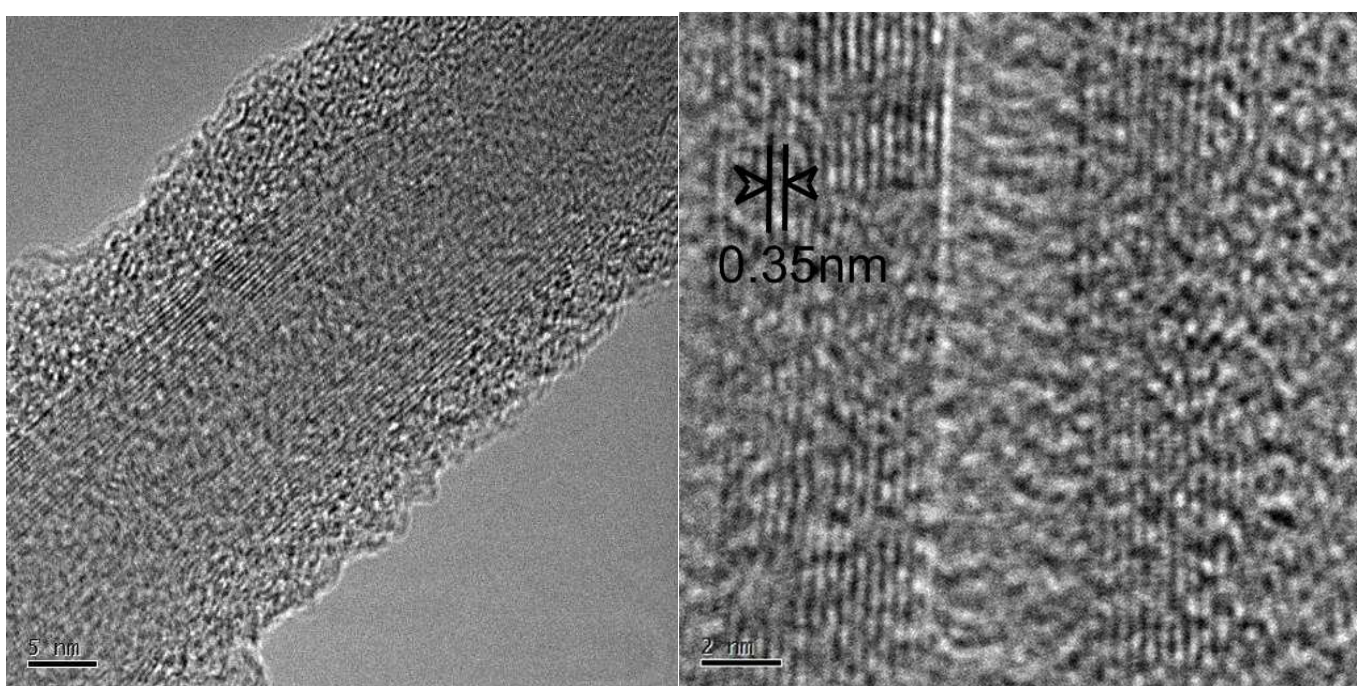

Figure 1: HRTEM images of solution formed nanofibers with the stacking parallel to the fiber length.

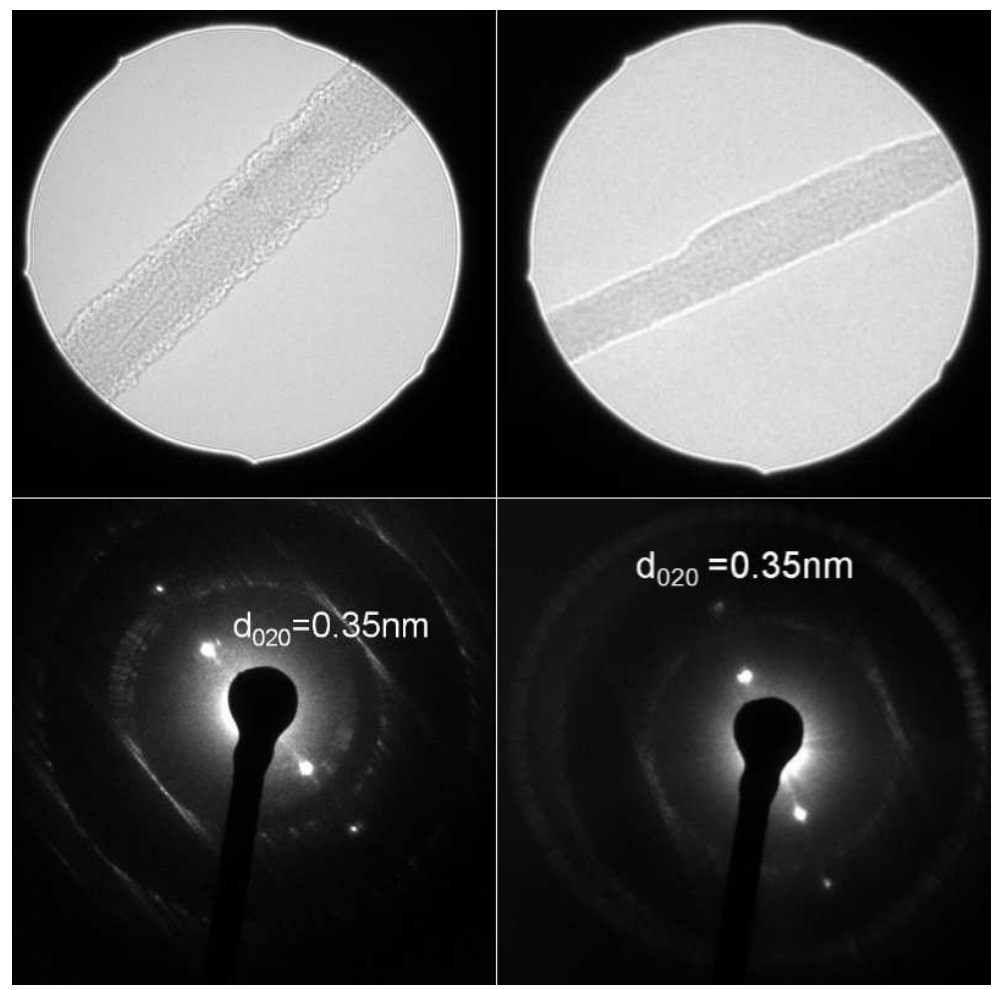

Figure 2: SAD diffraction images and patterns of parallel stacked fibers showing the (020) reflection, confirming parallel stacking of the polymer chains and the chain to chain spacing observed in the HRTEM images. 